\title{
Multigranulations Rough Set Method of Attribute Reduction in Information Systems Based on Evidence Theory
}

\author{
Minlun Yan \\ Department of Mathematics and Applied Mathematics, Lianyungang Teachers College, Lianyungang 222006, China \\ Correspondence should be addressed to Minlun Yan; haitouyan@163.com
}

Received 4 February 2014; Revised 16 June 2014; Accepted 17 June 2014; Published 1 July 2014

Academic Editor: Xiaojing Yang

Copyright ( 2014 Minlun Yan. This is an open access article distributed under the Creative Commons Attribution License, which permits unrestricted use, distribution, and reproduction in any medium, provided the original work is properly cited.

\begin{abstract}
Attribute reduction is one of the most important problems in rough set theory. However, from the granular computing point of view, the classical rough set theory is based on a single granulation. It is necessary to study the issue of attribute reduction based on multigranulations rough set. To acquire brief decision rules from information systems, this paper firstly investigates attribute reductions by combining the multigranulations rough set together with evidence theory. Concepts of belief and plausibility consistent set are proposed, and some important properties are addressed by the view of the optimistic and pessimistic multigranulations rough set. What is more, the multigranulations method of the belief and plausibility reductions is constructed in the paper. It is proved that a set is an optimistic (pessimistic) belief reduction if and only if it is an optimistic (pessimistic) lower approximation reduction, and a set is an optimistic (pessimistic) plausibility reduction if and only if it is an optimistic (pessimistic) upper approximation reduction.
\end{abstract}

\section{Introduction}

Rough set theory, originated by Pawlak in the early 1980s $[1,2]$, is an extension of the classical set theory and can be regarded as a soft computing tool to handle imprecision, vagueness, and uncertainty in date analysis. The theory has been found successful in applications, especially in the field of pattern recognition [3], medical diagnosis [4], data mining $[5,6]$, conflict analysis [7], algebra [8,9], and other fields $[10,11]$. Recently, the theory has generated a great deal of interest among more and more researchers.

Recently, several extensions of the rough set model have been proposed in terms of various requirements, such as the variable precision rough set (VPRS) model [12], the Bayesian rough set model [13], the fuzzy rough set model, and the rough fuzzy set model [14-16]. Equivalence relation is a basic notion in Pawlak's rough set model. The equivalence classes are employed to construct the lower and upper approximations of an arbitrary subset of the universe of discourse. However, the equivalence relation is a very restrictive condition that may limit applications of rough set. Hence, a variety of extensions of Pawlak's rough set were proposed by employing a more general mathematical concept, for example, arbitrary binary relations [17-19], neighborhood systems and Boolean algebras [20,21], and partitions and coverings of the universe of discourse $[22,23]$. In the view of granular computing (proposed by Zadeh [24]), a general concept described by a set is always characterized via the so-called lower and upper approximations under a single granulation; that is, the concept is depicted by known knowledge induced from a single relation (such as equivalence relation, tolerance relation, and reflexive relation) on the universe.

Since each set of the information granules can be considered as a granulation space, then one can call two or more than two information granules as multigranulations space. To make it more widely to apply the rough set theory in practical applications, Qian and Liang extended Pawlak's single-granulation rough set model to a multigranulations rough set model [25]. Multigranulations rough set was initially proposed by Qian and Liang [25], and later many researchers have extended the multigranulations rough set to the generalized multigranulations rough set. $\mathrm{Xu}$ et al. developed a variable multigranulations rough set model [26], a fuzzy multigranulations rough set model [27], a generalized 
multigranulations rough set approach [28], and a multigranulations rough set model in ordered information systems [29]. Yang et al. proposed the hierarchical structure properties of the multigranulations rough set [30-33] and multigranulations rough set in incomplete information system [34]. Lin et al. presented a neighborhood-based multigranulations rough set [35]. Qian et al. discussed the decision-theoretic rough set theory based on Bayesian decision procedure into the multigranulations [36]. She and He explored the topological structures and the properties of multigranulations rough set [37] and many others [38, 39].

Another important method used to deal with uncertainty in information systems is the Dempster-Shafer theory of evidence. It was originated by Dempster's concept of lower and upper probabilities [40] and extended by Shafer as a theory [41]. The basic representational structure in the theory is a belief structure, which can derive dual pairs of belief and plausibility functions. Subsequently, Zadeh generalized the Dempster-Shafer theory to the fuzzy environment based on his work on the concepts of information granularity [42] and the theory of possibility [43]. So as to evaluate the degrees of belief in fuzzy events, many authors have enriched the Dempster-Shafer theory in different ways. Interested readers can refer to [44] for a summary of some of these generalizations.

There are strong connections between rough set theory and Dempster-Shafer theory of evidence. It has been demonstrated that various belief structures are associated with various rough approximation spaces such that the different dual pairs of lower and upper approximation operators induced by rough approximation spaces may be used to interpret the corresponding dual pairs of belief and plausibility functions induced by belief structures [45-48].

It is well known that attribute reduction is one of the hot research topics of rough set theory. There are many attributes in information system in general. But some attributes are not always needed based on the various reasons. Several kinds of attribute reductions such as upper approximation reductions, lower approximation reductions, and positive region reductions were discussed in a decision system according to different requirements [49-51]. By now much study on this area had been reported and many useful results were obtained [50, 52-55]. While in our real life, we may face some problems in which the existing reductions cannot be disposed, on this situation, some new reductions are needed. In this paper, we attempt to investigate attribute reduction in multigranulations rough set based on evidence theory and its strong relations with existing reductions.

The organization of the rest of this paper is as follows. In Section 2, we give some basic concepts of information systems and Pawlak's rough set, multigranulations rough set, and evidence theory. In Section 3, evidence theory in optimistic multigranulations rough set and pessimistic multigranulations rough set has been constructed, and some important properties are discussed. In Section 4, we introduce the optimistic and pessimistic multigranulations rough set method of belief and plausibility reductions; then we also combine the belief structure with the lower and upper approximations. And in Section 5, we conclude the paper with a summary and outlook for further research.

\section{Preliminaries}

Throughout this paper, we assume that the universe is a nonempty finite set.

An information system is an order triple $S=(U, \mathrm{AT}, f)$, where $U=\left\{x_{1}, x_{2}, \ldots, x_{n}\right\}$ is a nonempty finite set of objects, $\mathrm{AT}=\left\{a_{1}, a_{2}, \ldots, a_{m}\right\}$ is a nonempty finite set of conditional attributes, and for any $a_{i} \in \mathrm{AT}, f_{a_{i}}: U \rightarrow V_{a_{i}}$ is a map, where $V_{a_{i}}$ is the domain of the attribute $a_{i}$. In particular, a target information system is given by $S=(U$, AT, $f, D, g)$, where $D=\left\{d_{1}, d_{2}, \ldots, d_{p}\right\}$ is a nonempty finite set of decision attributes, and for any $d_{j} \in D, g_{d_{j}}: U \rightarrow V_{d_{j}}$ is a map, where $V_{d_{j}}$ is the domain of the attribute $d_{j}$. In general, a target information system is consistent, if the partitions induced from the set of condition attributes AT are finer than the partitions induced from the set of decision attributes $D$. Otherwise, it is inconsistent.

For an information system, any attribute domain $V_{a}$ may contain special symbol "*” to represent that the value of an attribute is unknown. Here, we assume that an object $x \in U$ possesses only one value for an attribute $a, a \in$ AT. Thus, if the value of an attribute $a$ is missing, then the attribute value is the symbol " $*$ ", and the real value of the attribute must be from the set $V_{a} \backslash\{*\}$. Any domain value different from “*” will be called regular. A system in which values of all attributes for all objects from $U$ are regular (known) is called complete; otherwise it is called incomplete $[56,57]$.

Throughout this paper, we assume that the information system is the complete information system.

Suppose that $S=(U, \mathrm{AT}, f)$ is an information system, $R_{A}=\left\{(x, y) \mid f_{a_{i}}(x)=f_{a_{i}}(y), \forall a_{i} \in A\right\}$; let $U / R_{A}$ be a partition of $U$ induced by the attribute subset $A \subseteq$ AT. For any $x \in U,[x]_{A}=\left\{y \mid(x, y) \in R_{A}\right\}$; more information can be found in [58-60].

In the multigranulations rough set model, unlike Pawlak's rough set theory, the target concept is approximated via multiple partitions induced by multiple equivalence relations. Suppose that $S=(U, \mathrm{AT}, f)$ is an information system, $A_{1}, A_{2}, \ldots, A_{s}\left(s \leq 2^{|\mathrm{AT}|}\right)$ are attribute subsets, and $X \subseteq U$. Then the optimistic multigranulations lower approximation and upper approximation of $X$ related to $A_{1}, A_{2}, \ldots, A_{s}$ in $U$ are defined as follows:

$$
\begin{gathered}
\overline{\mathrm{OM}_{\sum_{i=1}^{s} A_{i}}}(X)=\left\{x \mid \vee\left([x]_{A_{i}} \subseteq X\right)\right\}, \\
\overline{\mathrm{OM}_{\sum_{i=1}^{s} A_{i}}}(X)=\left\{x \mid \wedge\left([x]_{A_{i}} \cap X \neq \emptyset\right)\right\} .
\end{gathered}
$$

And the pessimistic multigranulations lower approximation and upper approximation of $X$ related to $A_{1}, A_{2}, \ldots, A_{s}$ in $U$ are defined as follows:

$$
\begin{gathered}
\frac{\mathrm{PM}_{\sum_{i=1}^{s} A_{i}}}{}(X)=\left\{x \mid \wedge\left([x]_{A_{i}} \subseteq X\right)\right\}, \\
\overline{\mathrm{PM}_{\sum_{i=1}^{s} A_{i}}}(X)=\left\{x \mid \vee\left([x]_{A_{i}} \cap X \neq \emptyset\right)\right\} .
\end{gathered}
$$

More detailed introductions can be seen in $[58,59,61,62]$. 
In evidence theory [40, 41], a mass function of a universe $U$ can be defined by a map $m: P(U) \rightarrow[0,1]$, and this mass function satisfies two axioms:

$$
\begin{aligned}
& (M 1) \quad m(\emptyset)=0, \\
& (M 2) \quad \sum_{X \subseteq U} m(X)=1 .
\end{aligned}
$$

The value $m(X)$ represents the degree of belief that a specific element of $U$ belongs to set $X$ but not to any particular subset of $X$.

If $m(X)>0$, then $X$ is called a focal element. The family of all focal elements of $m$ are denoted by $M$. Then the pair $(M, m)$ is called a belief structure.

In information systems, each belief structure can derive a pair of belief and plausibility functions based on classical equivalence relation.

Definition 1 (see $[40,41])$. Let $(M, m)$ be a belief structure. A set function Bel : $P(U) \rightarrow[0,1]$ is referred to as a belief function on $U$, if for any $X \in P(U)$,

$$
\operatorname{Bel}(X)=\sum_{Y \subseteq X} m(Y) .
$$

A set function $\mathrm{Pl}: P(U) \rightarrow[0,1]$ is referred to as a plausibility function on $U$, if for any $X \in P(U)$,

$$
\operatorname{Pl}(X)=\sum_{Y \cap X \neq \emptyset} m(Y) .
$$

Remark 2. The above definition about belief and plausibility functions can also be defined as follows.

A set function Bel :P(U) $\rightarrow[0,1]$ is referred to as a belief function on $U$, if it satisfies the following three axioms [48]:

(1) $\operatorname{Bel}(\emptyset)=0$,

(2) $\operatorname{Bel}(U)=1$,

(3) for any positive integer $n$ and every collection $X_{1}, X_{2}, \ldots, X_{n} \subseteq U$,

$$
\operatorname{Bel}\left(\bigcup_{i=1}^{n} X_{i}\right) \geq \sum_{\emptyset \neq J \subseteq\{1,2, \ldots, n\}}(-1)^{|J|+1} \operatorname{Bel}\left(\bigcap_{i \in J} X_{i}\right) .
$$

A set function $\mathrm{Pl}: P(U) \rightarrow[0,1]$ is referred to as a plausibility function on $U$, if it satisfies the following three axioms [48]:

(1) $\operatorname{Pl}(\emptyset)=0$,

(2) $\operatorname{Pl}(U)=1$,

(3) for any positive integer $n$ and every collection $X_{1}, X_{2}, \ldots, X_{n} \subseteq U$,

$$
\operatorname{Pl}\left(\bigcap_{i=1}^{n} X_{i}\right) \leq \sum_{\emptyset \neq J \subseteq\{1,2, \ldots, n\}}(-1)^{|J|+1} \operatorname{Pl}\left(\bigcup_{i \in J} X_{i}\right) .
$$

From the definition and remark above, one can test and verify belief and plausibility functions by validating the three axioms above, respectively.

\section{Evidence Theory in Multigranulations Rough Set}

In this section, we will introduce the evidence theory in the optimistic multigranulations rough set and pessimistic multigranulations rough set and discuss some important properties of evidence theory in information system.

Definition 3. Let $S=(U, \mathrm{AT}, f)$ be an information system, $A_{1}, A_{2}, \ldots, A_{s} \subseteq \mathrm{AT}\left(s \leq 2^{|\mathrm{AT}|}\right)$, and let $R_{i}$ be the equivalence relation associated with $A_{i}$. For any $X \in U / R_{i}$, a mass function $m_{i}$ of $S$ can be defined as $m_{i}(X)=|X| /|U|$, where $|X|$ denotes the cardinality of a set $X$.

By the above definition, we can easily find that a mass function of information system satisfies two basic axioms. That is to say, for any $X \in U / R_{i}$ in information system, the following two axioms hold directly:

$$
\begin{array}{ll}
(M 1) & m_{i}(\emptyset)=0, \\
(M 2) & \sum_{X \subseteq U / R_{i}} m_{i}(X)=1 .
\end{array}
$$

Similarly, the family of all focal elements of $m$ is denoted by $M$ in optimistic multigranulations rough set. The pair $(M, m)$ is called a belief structure of the optimistic multigranulations rough set in information system; a pair of belief and plausibility function in the optimistic multigranulations rough set can be derived immediately.

Definition 4. Let $S=(U, \mathrm{AT}, f)$ be an information system and $(M, m)$ a belief structure.

(1) A set function Bel :P(U) $\rightarrow[0,1]$ is referred to as an optimistic belief function on $U$, if for any $X \in P(U)$,

$$
\operatorname{Bel}_{\sum_{i=1}^{s} A_{i}}^{o}(X)=\sum_{Y \subseteq X, Y \in U / R_{A_{i}}\left(\exists A_{i} \subseteq A T\right)} m_{i}(Y) .
$$

A set function $\mathrm{Pl}: P(U) \rightarrow[0,1]$ is referred to as an optimistic plausibility function on $U$, if for any $X \in P(U)$,

$$
\mathrm{Pl}_{\sum_{i=1}^{s} A_{i}}^{o}(X)=\sum_{Y \cap X \neq \emptyset, Y \in U / R_{A_{i}}\left(\forall A_{i} \subseteq A T\right)} m_{i}(Y) .
$$

(2) A set function Bel :P(U) $\rightarrow[0,1]$ is referred to as a pessimistic belief function on $U$, if for any $X \in P(U)$,

$$
\operatorname{Bel}_{\sum_{i=1}^{s} A_{i}}^{P}(X)=\sum_{Y \subseteq X, Y \in U / R_{A_{i}}\left(\forall A_{i} \subseteq A \mathrm{AT}\right)} m_{i}(Y) .
$$

A set function $\mathrm{Pl}: P(U) \rightarrow[0,1]$ is referred to as a pessimistic plausibility function on $U$, if for any $X \in P(U)$,

$$
\operatorname{Pl}_{\sum_{i=1}^{s} A_{i}}^{P}(X)=\sum_{Y \cap X \neq \emptyset, Y \in U / R_{A_{i}}\left(\exists A_{i} \subseteq A T\right)} m_{i}(Y) .
$$


Theorem 5. Let $S=(U, A T, f)$ be an information system, for any $X \subseteq U, A_{1}, A_{2}, \ldots, A_{s} \subseteq A T\left(s \leq 2^{|A T|}\right)$, denoted by

$$
\begin{aligned}
& B e l_{\sum_{i=1}^{s} A_{i}}^{o}(X)=\frac{\left|O M_{\sum_{i=1}^{s} A_{i}}(X)\right|}{|U|}, \\
& P l_{\sum_{i=1}^{s} A_{i}}^{o}(X)=\frac{\left|\overline{O M_{\sum_{i=1}^{s} A_{i}}}(X)\right|}{|U|}, \\
& \operatorname{Bel}_{\sum_{i=1}^{s} A_{i}}^{P}(X)=\frac{\left|P M_{\sum_{i=1}^{s} A_{i}}(X)\right|}{|U|}, \\
& P l_{\sum_{i=1}^{s} A_{i}}^{P}(X)=\frac{\left|\overline{P M_{\sum_{i=1}^{s} A_{i}}}(X)\right|}{|U|} .
\end{aligned}
$$

\section{Then}

$B e l_{\sum_{i=1}^{s} A_{i}}^{o}(X)$ is the optimistic belief function and $P l_{\sum_{i=1}^{s} A_{i}}^{o}(X)$ is the optimistic plausibility function of $U$;

$B e \sum_{\sum_{i=1}^{s} A_{i}}^{P}(X)$ is the pessimistic belief function and $P P_{\sum_{i=1}^{s} A_{i}}^{P}(X)$ is the pessimistic plausibility function of $U$.

Proof. We only prove $\operatorname{Bel}_{\sum_{i=1}^{s} A_{i}}^{o}(X)$ is the optimistic belief function of $U$; then analogously we can prove $\mathrm{Pl}_{\sum_{i=1}^{s} A_{i}}^{o}(X)$ is the optimistic plausibility function of $U$. Consider

(1) $\mathrm{Bel}_{\sum_{i=1}^{s} A_{i}}^{o}(\emptyset)=\left|\mathrm{OM}_{\sum_{i=1}^{s} A_{i}}(\emptyset)\right| /|U|=0 /|U|=0$,

(2) $\operatorname{Be}_{\sum_{i=1}^{s} A_{i}}^{o}(U)=\left|\mathrm{OM}_{\sum_{i=1}^{s} A_{i}}(U)\right| /|U|=|U| /|U|=1$,

(3) for any positive integer $n$ and every collection $X_{1}, X_{2}, \ldots, X_{n} \subseteq U$, we have

$\operatorname{Bel}_{\sum_{i=1}^{s} A_{i}}^{o}\left(X_{1} \cup X_{2} \cup \cdots \cup X_{n}\right)$

$=\frac{\left|\mathrm{OM}_{\sum_{i=1}^{s} A_{i}}\left(X_{1} \cup X_{2} \cup \cdots \cup X_{n}\right)\right|}{|U|}$

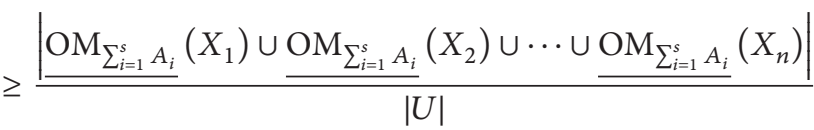

$=\sum_{j=1}^{n} \frac{\left|\mathrm{OM}_{\sum_{i=1}^{s} A_{i}}\left(X_{j}\right)\right|}{|U|}$

$-\sum_{k<j} \frac{\left|\mathrm{OM}_{\sum_{i=1}^{s} A_{i}}\left(X_{k}\right) \cap \underline{\mathrm{OM}_{\sum_{i=1}^{s} A_{i}}}\left(X_{j}\right)\right|}{|U|}+\cdots+(-1)^{n+1}$

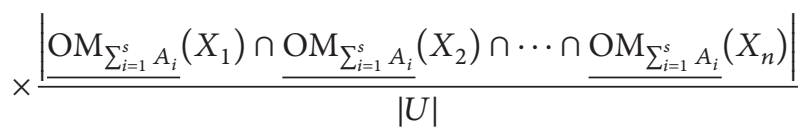

$$
\begin{aligned}
\geq & \sum_{j=1}^{n} \frac{\left|\mathrm{OM}_{\sum_{i=1}^{s} A_{i}}\left(X_{j}\right)\right|}{|U|}-\sum_{k<j} \frac{\left|\mathrm{OM}_{\sum_{i=1}^{s} A_{i}}\left(X_{k} \cap X_{j}\right)\right|}{|U|} \\
& +\cdots+(-1)^{n+1} \frac{\left|\mathrm{OM}_{\sum_{i=1}^{s} A_{i}}\left(X_{1} \cap X_{2} \cdots \cap X_{n}\right)\right|}{|U|} \\
= & \sum_{j=1}^{n} \operatorname{Bel}_{\sum_{i=1}^{s} A_{i}}^{o}\left(X_{j}\right)-\sum_{k<j} \operatorname{Bel}_{\sum_{i=1}^{s} A_{i}}^{o}\left(X_{k} \cap X_{j}\right) \\
& +\cdots+(-1)^{n+1} \operatorname{Bel}_{\sum_{i=1}^{s} A_{i}}^{o}\left(X_{1} \cap X_{2} \cap \cdots \cap X_{n}\right) .
\end{aligned}
$$

Similarly we can prove that $\operatorname{Bel}_{\sum_{i=1}^{s} A_{i}}^{P}(X)$ is the pessimistic belief function and $\operatorname{Pl}_{\sum_{i=1}^{s} A_{i}}^{P}(X)$ is the pessimistic plausibility function of $U$.

Thus, the theorem is proved.

From the theorem above, one can get the following properties of the belief function and plausibility function in the optimistic and pessimistic multigranulations rough set.

Theorem 6. Belief function and plausibility function based on the same belief structure are connected by the dual property in the optimistic multigranulations rough set and the pessimistic multigranulations rough set, respectively,

(1) $B e l_{\sum_{i=1}^{s} A_{i}}^{o}(X)=1-P l_{\sum_{i=1}^{s} A_{i}}^{o}(\sim X)$,

(2) $B e l_{\sum_{i=1}^{s} A_{i}}^{P}(X)=1-P l_{\sum_{i=1}^{s} A_{i}}^{P}(\sim X)$.

Proof. (1) It is clear that $\mathrm{OM}_{\sum_{i=1}^{s} A_{i}}(X)=\sim \overline{\mathrm{OM}_{\sum_{i=1}^{s} A_{i}}}(\sim X)$, so $\frac{\mid \mathrm{OM}_{\sum_{i=1}^{s} A_{i}}}{\text { Then }}(X)|=| U|-| \overline{\overline{\mathrm{OM}_{\sum_{i=1}^{s} A_{i}}}}(\sim X) \mid$.

$$
\begin{aligned}
\frac{\left|\mathrm{OM}_{\sum_{i=1}^{s} A_{i}}(X)\right|}{|U|} & =\frac{|U|-\left|\overline{\mathrm{OM}_{\sum_{i=1}^{s} A_{i}}}(\sim X)\right|}{|U|} \\
& =1-\frac{\left|\overline{\mathrm{OM}_{\sum_{i=1}^{s} A_{i}}}(\sim X)\right|}{|U|} .
\end{aligned}
$$

That is to say

$$
\mathrm{Bel}_{\sum_{i=1}^{s} A_{i}}^{o}(X)=1-\mathrm{Pl}_{\sum_{i=1}^{s} A_{i}}^{o}(\sim X) .
$$

(2) One can prove $\operatorname{Bel}_{\sum_{i=1}^{s} A_{i}}^{P}(X)=1-\operatorname{Pl}_{\sum_{i=1}^{s} A_{i}}^{P}(\sim X)$ to be similar.

Corollary 7. Let $S=(U, A T, f)$ be an information system, for any $X \subseteq U, A_{1}, A_{2}, \ldots, A_{s} \subseteq A T\left(s \leq 2^{|A T|}\right)$; then

(1) $B e l_{A_{i}}^{o}(X) \leq B e l_{\sum_{1 \leq j \leq s}^{i \neq j} A_{i}}^{o}(X) \leq B e l_{\sum_{i=1}^{s} A_{i}}^{o}(X) \leq$ $|X| /|U| \leq P l_{\sum_{i=1}^{s} A_{i}}^{o}(X) \leq P l_{\sum_{1 \leq j \leq s}^{i \neq j} A_{i}}^{o}(X) \leq P l_{A_{i}}^{o}(X)$,

(2) $\mathrm{Bel}_{\sum_{i=1}^{s} A_{i}}^{P}(X) \leq B e l_{\sum_{1<j \leq s}^{i \neq j} A_{i}}^{P}(X) \leq B e l_{A_{i}}^{P}(X) \leq$ $|X| /|U| \leq P l_{A_{i}}^{P}(X) \leq P l_{\sum_{1 \leq j \leq s}^{i \neq j} A_{i}}^{P}(X) \leq P l_{\sum_{i=1}^{s} A_{i}}^{P}(X)$. 
Proof. It can be obtained directly by combining the properties of the optimistic multigranulations rough set, pessimistic multigranulations rough set, and Theorem 5.

The difference between (1) and (2) in Corollary 7 is mainly because of the difference between the definition of the optimistic and pessimistic multigranulations rough set lower and upper approximations.

Example 8. Table 1 depicts a target information system containing some information about an emporium investment project. Locus, Investment, and Population density are the conditional attributes of the system, and Decision is the decision attribute. (In the sequel, $L, I, P$, and $D$ will stand for Locus, Investment, Population density, and Decision, resp.) The attribute domains are as follows: $V_{L}=$ \{Good, Common, Bad $\}, V_{I}=\{$ High, Low $\}, V_{P}=$ $\{$ Big, Small, Medium $\}$, and $V_{D}=\{$ Yes, No $\}$.

Let $X=\left\{x_{1}, x_{2}, x_{6}, x_{8}\right\}$; we have gotten

$$
\begin{gathered}
\underline{\mathrm{OM}_{L}}(X)=\underline{R_{L}}(X)=\left\{x_{8}\right\}, \\
\underline{\mathrm{PM}_{L}}(X)=\underline{R_{L}}(X)=\left\{x_{8}\right\}, \\
\overline{\mathrm{OM}_{L}}(X)=\overline{R_{L}}(X)=\left\{x_{1}, x_{2}, \ldots, x_{8}\right\}, \\
\overline{\mathrm{PM}_{L}}(X)=\overline{R_{L}}(X)=\left\{x_{1}, x_{2}, \ldots, x_{8}\right\}, \\
\underline{\mathrm{OM}_{L+P}}(X)=\left\{x_{1}, x_{2}, x_{8}\right\}, \quad \underline{\mathrm{PM}_{L+P}}(X)=\emptyset, \\
\overline{\mathrm{OM}_{L+P}}(X)=\left\{x_{1}, x_{2}, x_{6}, x_{7}, x_{8}\right\}, \\
\overline{\mathrm{OM}_{L+I+P}}(X)=\left\{x_{1}, x_{2}, \ldots, x_{8}\right\}, \\
\overline{\mathrm{OM}_{L+I+P}}(X)=\left\{x_{1}, x_{2}, x_{8}\right\}, \quad \underline{\mathrm{PM}_{L+I+P}}(X)=\emptyset, \\
\overline{\mathrm{PM}_{L+I+P}}(X)=\left\{x_{1}, x_{2}, \ldots, x_{7}, x_{8}\right\},
\end{gathered}
$$

So, we can calculate

$$
\begin{aligned}
& \operatorname{Bel}_{L}^{o}(X)=\frac{\left|\mathrm{OM}_{L}(X)\right|}{|U|}=\frac{1}{8}, \\
& \operatorname{Bel}_{L}^{P}(X)=\frac{\left|\mathrm{PM}_{L}(X)\right|}{|U|}=\frac{1}{8}, \\
& \mathrm{Pl}_{L}^{o}(X)=\frac{\left|\overline{\mathrm{OM}_{L}}(X)\right|}{|U|}=1, \\
& \operatorname{Pl}_{L}^{P}(X)=\frac{\left|\overline{\operatorname{PM}_{L}}(X)\right|}{|U|}=1, \\
& \operatorname{Bel}_{L+P}^{o}(X)=\frac{\left|\mathrm{OM}_{L+P}(X)\right|}{|U|}=\frac{3}{8}, \\
& \operatorname{Bel}_{L+P}^{P}(X)=\frac{\left|\mathrm{PM}_{L+P}(X)\right|}{|U|}=0,
\end{aligned}
$$

$$
\begin{aligned}
& \mathrm{Pl}_{L+P}^{o}(X)=\frac{\left|\overline{\mathrm{OM}_{L+P}}(X)\right|}{|U|}=\frac{5}{8}, \\
& \mathrm{Pl}_{L+P}^{P}(X)=\frac{\left|\overline{\mathrm{PM}_{L+P}}(X)\right|}{|U|}=1, \\
& \operatorname{Bel}_{L+I+P}^{o}(X)=\frac{\left|\mathrm{OM}_{L+I+P}(X)\right|}{|U|}=\frac{3}{8}, \\
& \operatorname{Bel}_{L+I+P}^{P}(X)=\frac{\left|\mathrm{PM}_{L+I+P}(X)\right|}{|U|}=0, \\
& \mathrm{Pl}_{L+I+P}^{o}(X)=\frac{\left|\overline{O M_{L+I+P}}(X)\right|}{|U|}=\frac{5}{8} \text {, } \\
& \mathrm{Pl}_{L+I+P}^{P}(X)=\frac{\left|\overline{\mathrm{PM}_{L+I+P}}(X)\right|}{|U|}=1 .
\end{aligned}
$$

Hence, the following is obvious:

$$
\begin{aligned}
& \operatorname{Bel}_{L}^{o}(X) \leq \operatorname{Bel}_{L+P}^{o}(X) \leq \operatorname{Bel}_{L+I+P}^{o}(X) \\
& \leq \frac{|X|}{|U|} \leq \mathrm{Pl}_{L+I+P}^{o}(X) \leq \mathrm{Pl}_{L+P}^{o}(X) \leq \mathrm{Pl}_{L}^{o}(X), \\
& \operatorname{Bel}_{L+I+P}^{P}(X) \leq \operatorname{Bel}_{L+P}^{P}(X) \leq \operatorname{Bel}_{L}^{P}(X) \\
& \quad \leq \frac{|X|}{|U|} \leq \operatorname{Pl}_{L}^{P}(X) \leq \operatorname{Pl}_{L+P}^{P}(X) \leq \operatorname{Pl}_{L+I+P}^{P}(X) .
\end{aligned}
$$

\section{Attribute Reduction Based on Evidence Theory}

In this section, we consider the optimistic multiple granulation rough set and the pessimistic multigranulations rough set method of the attribute reductions by introducing the concepts of belief and plausibility reductions in information system and compare them with the existing reductions.

Let $S=(U, \mathrm{AT}, f, D, g)$ be a decision information system, $A \subseteq \mathrm{AT}$, and let $R_{A}$ and $R_{D}$ be the equivalence relations of $U$, which are induced by the conditional attribute set $A$ and the decision attribute set $D=\{d\}$, respectively; we denote

$$
\begin{gathered}
\frac{U}{R_{A}}=\left\{\left[x_{i}\right]_{A} \mid x_{i} \in U\right\}, \\
\frac{U}{R_{D}}=\left\{D_{1}, D_{2}, \ldots, D_{r}\right\}, \\
\bar{\eta}_{A}^{o}=\left(\overline{\overline{O M}_{\sum_{A_{i} \in A} A_{i}}}\left(D_{1}\right), \overline{\mathrm{OM}_{\sum_{A_{i} \in A} A_{i}}}\left(D_{2}\right),\right. \\
\left.\ldots, \overline{\mathrm{OM}_{\sum_{A_{i} \in A} A_{i}}}\left(D_{r}\right)\right),
\end{gathered}
$$


TABLE 1: A target information system about emporium investment project.

\begin{tabular}{lcccc}
\hline Project & Locus & Investment & Population density & Decision \\
\hline$x_{1}$ & Common & High & Big & Yes \\
$x_{2}$ & Bad & High & Big & Yes \\
$x_{3}$ & Bad & Low & Small & No \\
$x_{4}$ & Bad & Low & Small & No \\
$x_{5}$ & Bad & Low & Small & Yes \\
$x_{6}$ & Bad & High & Medium & No \\
$x_{7}$ & Common & High & Medium & Yes \\
$x_{8}$ & Good & High & Medium & \\
\hline
\end{tabular}

$$
\begin{aligned}
& \underline{\eta}_{A}^{o}=\left(\underline{\mathrm{OM}_{\sum_{A_{i} \in A} A_{i}}}\left(D_{1}\right), \underline{\mathrm{OM}_{\sum_{A_{i} \in A} A_{i}}}\left(D_{2}\right),\right. \\
& \left.\ldots, \underline{\mathrm{OM}_{\sum_{A_{i} \in A} A_{i}}}\left(D_{r}\right)\right), \\
& \bar{\eta}_{A}^{P}=\left(\overline{\mathrm{PM}_{\sum_{A_{i} \in A} A_{i}}}\left(D_{1}\right), \overline{\mathrm{PM}_{\sum_{A_{i} \in A} A_{i}}}\left(D_{2}\right),\right. \\
& \left.\ldots, \overline{\mathrm{PM}_{\sum_{A_{i} \in A} A_{i}}}\left(D_{r}\right)\right) \text {, }
\end{aligned}
$$

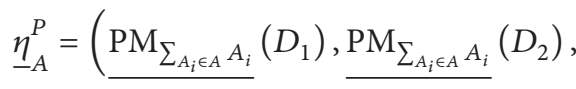

$$
\begin{aligned}
& \left.\ldots, \underline{\mathrm{PM}_{\sum_{A_{i} \in A} A_{i}}}\left(D_{r}\right)\right),
\end{aligned}
$$

where $\left[x_{i}\right]_{A}=\left\{y \in U \mid\left(x_{i}, y\right) \in R_{A}\right\}$.

Definition 9 (see [58]). Let $S=(U, \mathrm{AT}, f, D, g)$ be a decision information system in which $A \subseteq \mathrm{AT}$.

(1) If $\bar{\eta}_{A}^{o}=\bar{\eta}_{\mathrm{AT}}^{o}$, then $A$ is referred to as an optimistic upper approximation consistent set of $S$ with respect to the equivalence relation $R_{A}$; if $A$ is an optimistic upper approximation consistent set of $S$ and for any $A^{\prime} \subseteq A, A^{\prime}$ is not the optimistic upper approximation consistent set of $S$, then $A$ is referred to as an optimistic upper approximation reduction of $S$ with respect to the equivalence relation $R_{A}$.

(2) If $\underline{\eta}_{A}^{o}=\underline{\eta}_{\mathrm{AT}}^{o}$, then $A$ is referred to as an optimistic lower approximation consistent set of $S$ with respect to the equivalence relation $R_{A}$; if $A$ is an optimistic lower approximation consistent set of $S$ and for any $A^{\prime} \subseteq A, A^{\prime}$ is not the optimistic lower approximation consistent set of $S$, then $A$ is referred to as an optimistic lower approximation reduction of $S$ with respect to the equivalence relation $R_{A}$.

(3) If $\bar{\eta}_{A}^{P}=\bar{\eta}_{\mathrm{AT}}^{P}$, then $A$ is referred to as a pessimistic upper approximation consistent set of $S$ with respect to the equivalence relation $R_{A}$; if $A$ is a pessimistic upper approximation consistent set of $S$ and for any $A^{\prime} \subseteq A, A^{\prime}$ is not the pessimistic upper approximation consistent set of $S$, then $A$ is referred to as a pessimistic upper approximation reduction of $S$ with respect to the equivalence relation $R_{A}$.
(4) If $\underline{\eta}_{A}^{P}=\underline{\eta}_{\mathrm{AT}}^{P}$, then $A$ is referred to as a pessimistic lower approximation consistent set of $S$ with respect to the equivalence relation $R_{A}$; if $A$ is a pessimistic lower approximation consistent set of $S$ and for any $A^{\prime} \subseteq A, A^{\prime}$ is not the pessimistic lower approximation consistent set of $S$, then $A$ is referred to as a pessimistic lower approximation reduction of $S$ with respect to the equivalence relation $R_{A}$. From the definitions above, one can get the following theorem directly.

Theorem 10. Let $S=(U, A T, f, D, g)$ be a decision information system in which $A \subseteq A T$; then

(1) A is an optimistic upper approximation consistent set of $S$ if and only if, for any $D_{j} \in U / R_{D}, \overline{O M_{\sum_{A_{i} \in A} A_{i}}}\left(D_{j}\right)=$ $\overline{R_{A T}}\left(D_{j}\right)$ holds;

(2) A is an optimistic lower approximation consistent set of $S$ if and only if, for any $D_{j} \in U / R_{D}, O M_{\sum_{A_{i} \in A} A_{i}}\left(D_{j}\right)=$ $\underline{R_{A T}}\left(D_{j}\right)$ holds;

(3) A is a pessimistic upper approximation consistent set of $S$ if and only if, for any $D_{j} \in U / R_{D}, \overline{P M_{\sum_{A_{i} \in A} A_{i}}}\left(D_{j}\right)=$ $\overline{R_{A T}}\left(D_{j}\right)$ holds;

(4) $A$ is a pessimistic lower approximation consistent set of $S$ if and only if, for any $D_{j} \in U / R_{D}, P M_{\sum_{A_{i} \in A} A_{i}}\left(D_{j}\right)=$ $\underline{R_{A T}}\left(D_{j}\right)$ holds.

Proof. It can be derived easily from the definition of the optimistic upper and lower approximation reduction and the pessimistic upper and lower approximation reduction.

Definition 11. Let $S=(U, \mathrm{AT}, f, D, g)$ be a decision information system in which $A \subseteq \mathrm{AT}$.

(1) If for any $X \in U / R_{\mathrm{AT}}, \mathrm{Bel}_{\sum_{A_{i} \in A} A_{i}}(X)=\mathrm{Bel}_{\mathrm{AT}}^{o}(X)$, then $A$ is referred to as an optimistic belief consistent set of $S$; if $A$ is an optimistic belief consistent set of $S$ and for any $A^{\prime} \subseteq A, A^{\prime}$ is not the optimistic belief consistent set of $S$, then $A$ is referred to as an optimistic belief reduction of $S$.

(2) If for any $X \in U / R_{\mathrm{AT}}, \mathrm{Pl}_{\sum_{A_{i} \in A} A_{i}}^{o}(X)=\mathrm{Pl}_{\mathrm{AT}}^{o}(X)$, then $A$ is referred to as an optimistic plausibility consistent set of $S$; if $A$ is an optimistic plausibility consistent 
set of $S$ and for any $A^{\prime} \subseteq A, A^{\prime}$ is not the optimistic plausibility consistent set of $S$, then $A$ is referred to as an optimistic plausibility reduction of $S$.

(3) If for any $X \in U / R_{\mathrm{AT}}, \mathrm{Bel}_{\sum_{A_{i} \in A} A_{i}}^{P}(X)=\mathrm{Bel}_{\mathrm{AT}}^{P}(X)$, then $A$ is referred to as a pessimistic belief consistent set of $S$; if $A$ is a pessimistic belief consistent set of $S$ and for any $A^{\prime} \subseteq A, A^{\prime}$ is not the pessimistic belief consistent set of $S$, then $A$ is referred to as a pessimistic belief reduction of $S$.

(4) If for any $X \in U / R_{\mathrm{AT}}, \mathrm{Pl}_{\sum_{A_{i} \in \mathrm{A}} A_{i}}^{P}(X)=\mathrm{Pl}_{\mathrm{AT}}^{P}(X)$, then $A$ is referred to as a pessimistic plausibility consistent set of $S$; if $A$ is a pessimistic plausibility consistent set of $S$ and for any $A^{\prime} \subseteq A, A^{\prime}$ is not the pessimistic plausibility consistent set of $S$, then $A$ is referred to as a pessimistic plausibility reduction of $S$.

Theorem 12. Let $S=(U, A T, f, D, g)$ be a decision information system in which $A \subseteq A T$; then

(1) A is an optimistic belief consistent set of $S$ if and only if $A$ is an optimistic lower approximation consistent set of $S$;

(2) A is an optimistic plausibility consistent set of $S$ if and only if $A$ is an optimistic upper approximation consistent set of $S$;

(3) A is a pessimistic belief consistent set of $S$ if and only if $A$ is a pessimistic lower approximation consistent set of $S$;

(4) $A$ is a pessimistic plausibility consistent set of $S$ if and only if $A$ is a pessimistic upper approximation consistent set of $S$.

Proof. (1) Assume that $A$ is an optimistic belief consistent set of $S$; for any $X \in U / R_{\mathrm{AT}}$, we have $\operatorname{Bel}_{\sum_{A_{i} \in A} A_{i}}^{O}(X)=$ $\mathrm{Bel}_{\mathrm{AT}}^{o}(X)$. That is to say, $\left|\mathrm{OM}_{\sum_{A_{i} \in A} A_{i}}(X)\right|=\left|\underline{R_{\mathrm{AT}}}(X)\right|$. Then by the definition of the optimistic lower approximation we have $[x]_{\mathrm{AT}} \subseteq[x]_{A_{i}}$; then $\mathrm{OM}_{\sum_{A_{i} \in \mathrm{A}} A_{i}}(X) \subseteq \underline{R_{\mathrm{AT}}}(X)$. So $\mathrm{OM}_{\sum_{A_{i} \in A} A_{i}}(X)=\underline{R_{\mathrm{AT}}}(X)$. By Theorem 10 , we obtain $A$ is an optimistic lower approximation consistent set of $S$.

Conversely, if $A$ is an optimistic lower approximation consistent set of $S$, for any $D_{j} \in U / R_{D}$, we have $\mathrm{OM}_{\sum_{A_{i} \in A} A_{i}}\left(D_{j}\right)=\underline{R_{\mathrm{AT}}}\left(D_{j}\right)$. Then $\left|\mathrm{OM}_{\sum_{A_{i} \in A} A_{i}}\left(D_{j}\right)\right| /|U|=$

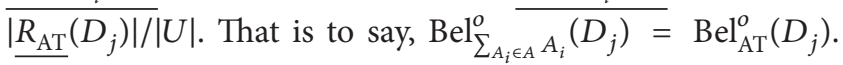
Thus $A$ is an optimistic belief consistent set of $S$.

(2) Assume that $A$ is an optimistic plausibility consistent set of $S$; for any $X \in U / R_{\mathrm{AT}}$, we have $\mathrm{Pl}_{\sum_{A_{i} \in A} A_{i}}(X)=\mathrm{Pl}_{\mathrm{AT}}^{O}(X)$. That is to say, $\left|\overline{\mathrm{OM}_{\sum_{A_{i} \in A} A_{i}}}(X)\right|=\left|\overline{R_{\mathrm{AT}}}(X)\right|$. By the definition of the optimistic upper approximation we have, $[x]_{\mathrm{AT}} \subseteq$ $[x]_{A_{i}}$. Then $\overline{\mathrm{OM}_{\sum_{A_{i} \in A} A_{i}}}(X) \supseteq \overline{R_{\mathrm{AT}}}(X)$. So $\overline{\mathrm{OM}_{\sum_{A_{i} \in A} A_{i}}}(X)=$ $\overline{R_{\mathrm{AT}}}(X)$. By Theorem 10 , we obtain $A$ is an optimistic upper approximation consistent set of $S$.

Conversely, if $A$ is an optimistic upper approximation consistent set of $S$, for any $D_{j} \in U / R_{D}$, we have
TABLE 2: A system about emporium investment project.

\begin{tabular}{lc}
\hline Project & Decision \\
\hline$x_{1}$ & Yes \\
$x_{2}$ & Yes \\
$x_{3}$ & No \\
$x_{4}$ & No \\
$x_{5}$ & No \\
$x_{6}$ & Yes \\
$x_{7}$ & No \\
$x_{8}$ & Yes \\
\hline
\end{tabular}

$\overline{\mathrm{OM}_{\sum_{A_{i} \in A} A_{i}}}\left(D_{j}\right)=\overline{R_{\mathrm{AT}}}\left(D_{j}\right)$. Then $\left|\overline{\mathrm{OM}_{\sum_{A_{i} \in A} A_{i}}}\left(D_{j}\right)\right| /|U|=$ $\left|\overline{R_{\mathrm{AT}}}\left(D_{j}\right)\right| /|U|$. That is to say, $\mathrm{Pl}_{\sum_{A_{i} \in A} A_{i}}\left(D_{j}\right)=\mathrm{Pl}_{\mathrm{AT}}^{o}\left(D_{j}\right)$. Thus $A$ is an optimistic plausibility consistent set of $S$.

(3) It is straightforward by (1).

(4) It is straightforward by (2).

Hence, the proof is completed.

Corollary 13. Let $S=(U, A T, f, D, g)$ be a decision information system in which $A \subseteq A T$; then

(1) $A$ is an optimistic belief reduction of $S$ if and only if $A$ is an optimistic lower approximation reduction of $S$;

(2) $A$ is an optimistic plausibility reduction of $S$ if and only if $A$ is an optimistic upper approximation reduction of $S$;

(3) $A$ is a pessimistic belief reduction of $S$ if and only if $A$ is a pessimistic lower approximation reduction of $S$;

(4) $A$ is a pessimistic plausibility reduction of $S$ if and only if $A$ is a pessimistic upper approximation reduction of $S$.

We have shown that the belief and plausibility reduction are the same with lower and upper approximation reduction. One may deal with some issue in which the lower and upper approximation reduction cannot be explained, while the belief and plausibility reduction can be well explained. Just like this, indefinite integral and definite integral are two basic problems in the integral calculus. Indefinite integral is the inverse operation of differentiation, while definite integral is the limit of a particular type of the sum. There are both difference and connection between them. The definite integral and the indefinite integral can be connected in theory using Newton-leibniz formula, while some functions still can be integrated, though they have no antiderivative on closed interval. Under this circumstance, indefinite integral cannot be evaluated by Newton-leibniz formula. Next we use an example to illustrate this problem.

Example 14. Table 2 depicts a system containing some information about an emporium investment project. All projects are $U=\left\{x_{1}, x_{2}, \ldots, x_{8}\right\}$, and Decision is the decision attribute. (In the sequel, $D$ will stand for Decision.)

In this example, there are no conditional attributes. So we cannot use the lower and upper approximations to deal with this system. Based on evidence theory, each object can be given corresponding confidence degree according to the 
existing information. And this confidence degree after normalization can be used as a new mass function. Then we can apply the evidence theory to dispose this system. Until now, we have not found a good method to evaluate the confidence degree. This will be an important issue of our research in the future.

\section{Conclusions}

It is well known that rough set theory has been regarded as a generalization of the classical set theory in some cases. In this paper, we have combined the rough set theory and evidence theory, in order to study the problem of attribute reductions. By introducing mass function based on the multigranulations rough set, we have considered the notions of the multigranulations rough set method of belief and plausibility reductions. What is more, we have found that a set is an optimistic (pessimistic) belief reduction if and only if it is an optimistic (pessimistic) lower approximation reduction, and a set is an optimistic (pessimistic) plausibility reduction if and only if it is an optimistic (pessimistic) upper approximation reduction by optimistic and pessimistic frames, respectively. And we will investigate the specific application of theories obtained in our further study.

\section{Conflict of Interests}

The author declares that there is no conflict of interests regarding the publication of this paper.

\section{References}

[1] Z. Pawlak, "Rough sets," International Journal of Computer and Information Sciences, vol. 11, no. 5, pp. 341-356, 1982.

[2] Z. Pawlak, Rough Set: Theoretical Aspects of Reasoning about Data, Kluwer Academic Publishers, Dordrecht, The Netherlands, 1991.

[3] R. W. Swiniarski and A. Skowron, "Rough set methods in feature selection and recognition," Pattern Recognition Letters, vol. 24, no. 6, pp. 833-849, 2003.

[4] S. Tsumoto, "Automated extraction of medical expert system rules from clinical databases based on rough set theory," Information Sciences, vol. 112, no. 1-4, pp. 67-84, 1998.

[5] V. S. Ananthanarayana, M. Narasimha Murty, and D. K. Subramanian, "Tree structure for efficient data mining using rough sets," Pattern Recognition Letters, vol. 24, no. 6, pp. 851-862, 2003.

[6] C. Chan, "A rough set approach to attribute generalization in data mining," Information Sciences, vol. 107, no. 1-4, pp. 169-176, 1998.

[7] Z. Pawlak, "Some remarks on conflict analysis," European Journal of Operational Research, vol. 166, no. 3, pp. 649-654, 2005.

[8] W. Cheng, Z. W. Mo, and J. Wang, "Notes on "the lower and upper approximations in a fuzzy group" and 'rough ideals in semigroups"' Information Sciences, vol. 177, no. 22, pp. 51345140, 2007.

[9] B. Davvaz and M. Mahdavipour, "Roughness in modules," Information Sciences, vol. 176, no. 24, pp. 3658-3674, 2006.
[10] I. Düntsch and G. Gediga, "Uncertainty measures of rough set prediction," Artificial Intelligence, vol. 106, no. 1, pp. 109-137, 1998.

[11] G. Jeon, D. Kim, and J. Jeong, "Rough sets attributes reduction based expert system in interlaced video sequences," IEEE Transactions on Consumer Electronics, vol. 52, no. 4, pp. 1348$1355,2006$.

[12] W. Ziarko, "Variable precision rough set model," Journal of Computer and System Sciences, vol. 46, no. 1, pp. 39-59, 1993.

[13] D. Ślęzak and W. Ziarko, "The investigation of the Bayesian rough set model," International Journal of Approximate Reasoning, vol. 40, no. 1-2, pp. 81-91, 2005.

[14] D. Dubois and H. Prade, "Rough fuzzy sets and fuzzy rough sets," International Journal of General Systems, vol. 17, no. 2-3, pp. 191-209, 1990.

[15] W. Wu, J. Mi, and W. Zhang, "Generalized fuzzy rough sets," Information Sciences, vol. 151, pp. 263-282, 2003.

[16] W.-Z. Wu and W.-X. Zhang, "Constructive and axiomatic approaches of fuzzy approximation operators," Information Sciences, vol. 159, no. 3-4, pp. 233-254, 2004.

[17] S. Greco, B. Matarazzo, and R. Slowinski, "Rough approximation by dominance relations," International Journal of Intelligent Systems, vol. 17, no. 2, pp. 153-171, 2002.

[18] R. Slowinski and D. Vanderpooten, "A generalized definition of rough approximations based on similarity," IEEE Transactions on Knowledge and Data Engineering, vol. 12, no. 2, pp. 331-336, 2000.

[19] Y. Y. Yao, "Constructive and algebraic methods of the theory of rough sets," Information Sciences, vol. 109, no. 1-4, pp. 21-47, 1998.

[20] D. Boixader, J. Jacas, and J. Recasens, "Upper and lower approximations of fuzzy sets," International Journal of General Systems, vol. 29, no. 4, pp. 555-568, 2000.

[21] W. Wu and W. Zhang, "Neighborhood operator systems and approximations," Information Sciences, vol. 144, no. 1-4, pp. 201217,2002

[22] C. Degang, W. Changzhong, and H. Qinghua, "A new approach to attribute reduction of consistent and inconsistent covering decision systems with covering rough sets," Information Sciences, vol. 177, no. 17, pp. 3500-3518, 2007.

[23] W. Zhu and F. Wang, "On three types of covering-based rough sets," IEEE Transactions on Knowledge and Data Engineering, vol. 19, no. 8, pp. 1131-1143, 2007.

[24] L. A. Zadeh, "Toward a theory of fuzzy information granulation and its centrality in human reasoning and fuzzy logic," Fuzzy Sets and Systems, vol. 90, no. 2, pp. 111-127, 1997.

[25] Y. H. Qian and J. Y. Liang, "Rough set method based on multigranulations," in Proceedings of the 5th IEEE International Conference on Cognitive Informatics (ICCI '06), pp. 297-304, Beijing, China, July 2006.

[26] W. H. Xu, X. T. Zhang, and Q. R. Wang, "A generalized multigranulation rough set approach," Lecture Notes in Computer Science, vol. 6840, pp. 681-689, 2012.

[27] W. H. Xu, Q. R. Wang, and X. T. Zhang, "Multi-granulation fuzzy rough sets in a fuzzy tolerance approximation space," International Journal of Fuzzy Systems, vol. 13, no. 4, pp. 246259, 2011.

[28] X. Wei-Hua, Z. Xiao-Yan, and Z. Wen-Xiu, "Knowledge granulation, knowledge entropy and knowledge uncertainty measure in ordered information systems," Applied Soft Computing, vol. 9, no. 4, pp. 1244-1251, 2009. 
[29] W. Xu, W. Sun, X. Zhang, and W. Zhang, "Multiple granulation rough set approach to ordered information systems," International Journal of General Systems, vol. 41, no. 5, pp. 475-501, 2012.

[30] X. B. Yang, Y. S. Qi, X. N. Song, and J. Y. Yang, "Test cost sensitive multigranulation rough set: model and minimal cost selection," Information Sciences, vol. 250, pp. 184-199, 2013.

[31] X. B. Yang, Y. H. Qian, and J. Y. Yang, "Hierarchical structures on multigranulation spaces," Journal of Computer Science and Technology, vol. 27, no. 6, pp. 1169-1183, 2012.

[32] X. B. Yang, Y. H. Qian, and J. Y. Yang, "On characterizing hierarchies of granulation structures via distances," Fundamenta Informaticae, vol. 123, no. 3, pp. 365-380, 2013.

[33] X. Yang, X. Song, Y. She, and J. Yang, "Hierarchy on multigranulation structures: a knowledge distance approach," International Journal of General Systems, vol. 42, no. 7, pp. 754-773, 2013.

[34] X. B. Yang, X. N. Song, Z. H. Chen, and J. Y. Yang, "Multigranulation rough sets in incomplete information system," in Incomplete Information System and Rough Set Theory, pp. 195-222, Springer, Berlin, Germany, 2012.

[35] G. Lin, Y. Qian, and J. Li, "NMGRS: neighborhood-based multigranulation rough sets," International Journal of Approximate Reasoning, vol. 53, no. 7, pp. 1080-1093, 2012.

[36] Y. H. Qian, H. Zhang, Y. L. Sang, and J. Y. Liang, "Multigranulation decision-theoretic rough sets," International Journal of Approximate Reasoning, vol. 55, pp. 225-237, 2014.

[37] Y. H. She and X. L. He, "On the structure of the multigranulation rough set model," Knowledge-Based Systems, vol. 36, pp. 81-92, 2012.

[38] W. Xu, W. Sun, and Y. Liu, "Optimistic multi-granulation fuzzy rough set model based on triangular norm," Lecture Notes in Computer Science, vol. 7414, pp. 20-27, 2012.

[39] W. H. Xu, W. X. Sun, and Y. F. Liu, "Optimistic multi-granulation fuzzy rough set model in ordered information system," in Proceedings of the IEEE International Conference on Granular Computing (GrC '12), pp. 563-568, Hangzhou, China, August 2012.

[40] A. P. Dempster, "Upper and lower probabilities induced by a multivalued mapping," Annals of Mathematical Statistics, vol. 38, pp. 325-339, 1967.

[41] G. Shafer, A Mathematical Theory of Evidence, Princeton University Press, Princeton, NJ, USA, 1976.

[42] L. A. Zadeh, "Fuzzy sets and information granularity," in Advances in Fuzzy Set Theory and Applications, M. Gupta, R. Ragade, and R. R. Yager, Eds., pp. 3-18, North-Holland, Amsterdam, The Netherlands, 1979.

[43] L. A. Zadeh, "Fuzzy sets as a basis for a theory of possibility," Fuzzy Sets and Systems, vol. 1, no. 1, pp. 3-28, 1978.

[44] W. Wu, Y. Leung, and J. Mi, "On generalized fuzzy belief functions in infinite spaces," IEEE Transactions on Fuzzy Systems, vol. 17, no. 2, pp. 385-397, 2009.

[45] A. Skowron and J. Graymala-Busse, "From rough set theory to evidence theory," in Advance in the Dempster-Shafer Theory of Evidence, RR. Yager, M. Fedrizzi, and J. Kacprzyk, Eds., pp. 193236, Wiley, New York, NY, USA, 1994.

[46] W. Z. Wu, Y. Leung, and W. X. Zhang, "Connections between rough set theory and Dempster-Shafer theory of evidence," International Journal of General Systems, vol. 31, no. 4, pp. 405430, 2002.
[47] W. Z. Wu, M. Zhang, H. Z. Li, and J. S. Mi, "Knowledge reduction in random information systems via Dempster-Shafer theory of evidence," Information Sciences, vol. 174, no. 3-4, pp. 143164, 2005.

[48] Y. Y. Yao and P. J. Lingras, "Interpretations of belief functions in the theory of rough sets," Information Sciences, vol. 104, no. 1-2, pp. 81-106, 1998.

[49] X. B. Yang and J. Y. Yang, Incomplete Information System and Rough Set Theory: Models an d Attribute Reductions, Science Press, Beijing, China, 2012.

[50] W. X. Zhang, J. S. Mi, and W. Z. Wu, "Approaches to knowledge reductions in inconsistent systems," International Journal of Intelligent Systems, vol. 21, no. 9, pp. 989-1000, 2003.

[51] W. X. Zhang, Y. Leung, and W. Z. Wu, Information Systems and Knowledge Discovery, Science Press, Beijing, China, 2004.

[52] M. Kryszkiewicz, "Comparative studies of alternative type of knowledge reduction in inconsistent systems," International Journal of Intelligent Systems, vol. 16, pp. 105-120, 2001.

[53] Y. Leung, W.-Z. Wu, and W.-X. Zhang, "Knowledge acquisition in incomplete information systems: a rough set approach," European Journal of Operational Research, vol. 168, no. 1, pp. 164-180, 2006.

[54] W.-H. Xu and W.-X. Zhang, "Measuring roughness of generalized rough sets induced by a covering," Fuzzy Sets and Systems, vol. 158, no. 22, pp. 2443-2455, 2007.

[55] W. X. Zhang, W. Z. Wu, J. Y. Liang, and D. Y. Li, Theory and Method of Rough Sets, Science Press, Beijing, China, 2001.

[56] Y. Leung and D. Li, "Maximal consistent block technique for rule acquisition in incomplete information systems," Information Sciences, vol. 153, pp. 85-106, 2003.

[57] J. Y. Liang and D. Y. Li, Uncertainty and Knowledge Acquisition in Information Systems, Science Press, Beijing, China, 2005.

[58] Y.-H. Qian, J.-Y. Liang, Y.-Y. Yao, and C.-H. Dang, "MGRS: a multi-granulation rough set," Information Sciences, vol. 180, no. 6, pp. 949-970, 2010.

[59] W. H. Xu, X. Y. Zhang, and W. X. Zhang, "Two new types of multi-granulation rough set," submitted to Information Sciences.

[60] W. X. Zhang, Y. Leung, and W. Z. Wu, "Fuzzy information systems and knowledge discovery," in Information Systems and Knowledge Discovery, pp. 96-112, Science Press, 2003.

[61] Y. H. Qian, J. Y. Liang, W. Pedrycz, and C. Y. Dang, "Positive approximation: an accelerator for attribute reduction in rough set theory," Artificial Intelligence, vol. 174, no. 9-10, pp. 597-618, 2010.

[62] Y. H. Qian, J. Y. Liang, and W. Wei, "Pessimistic rough decision," in Proceedings of the 2nd International Workshop on Rough Sets Theory, pp. 440-449, 2010. 


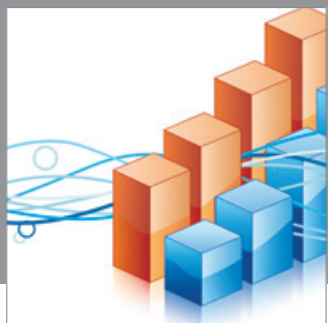

Advances in

Operations Research

mansans

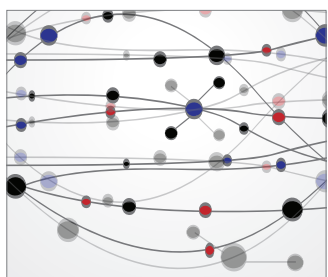

The Scientific World Journal
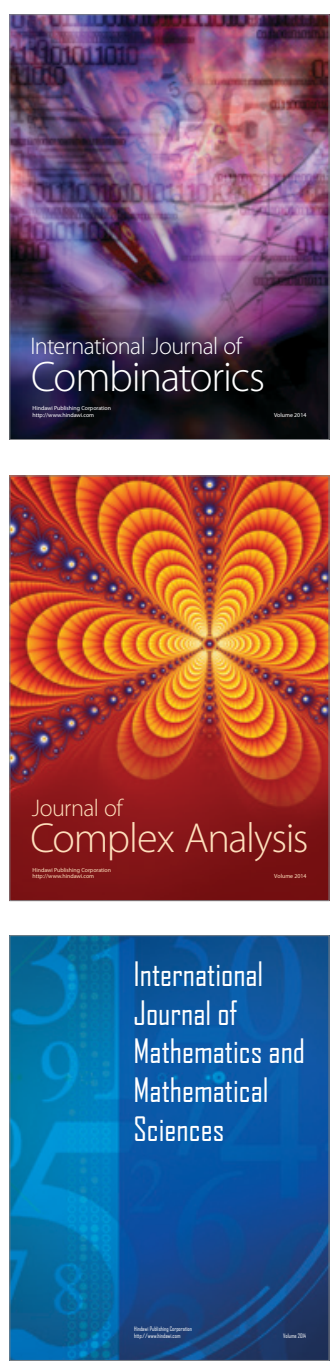
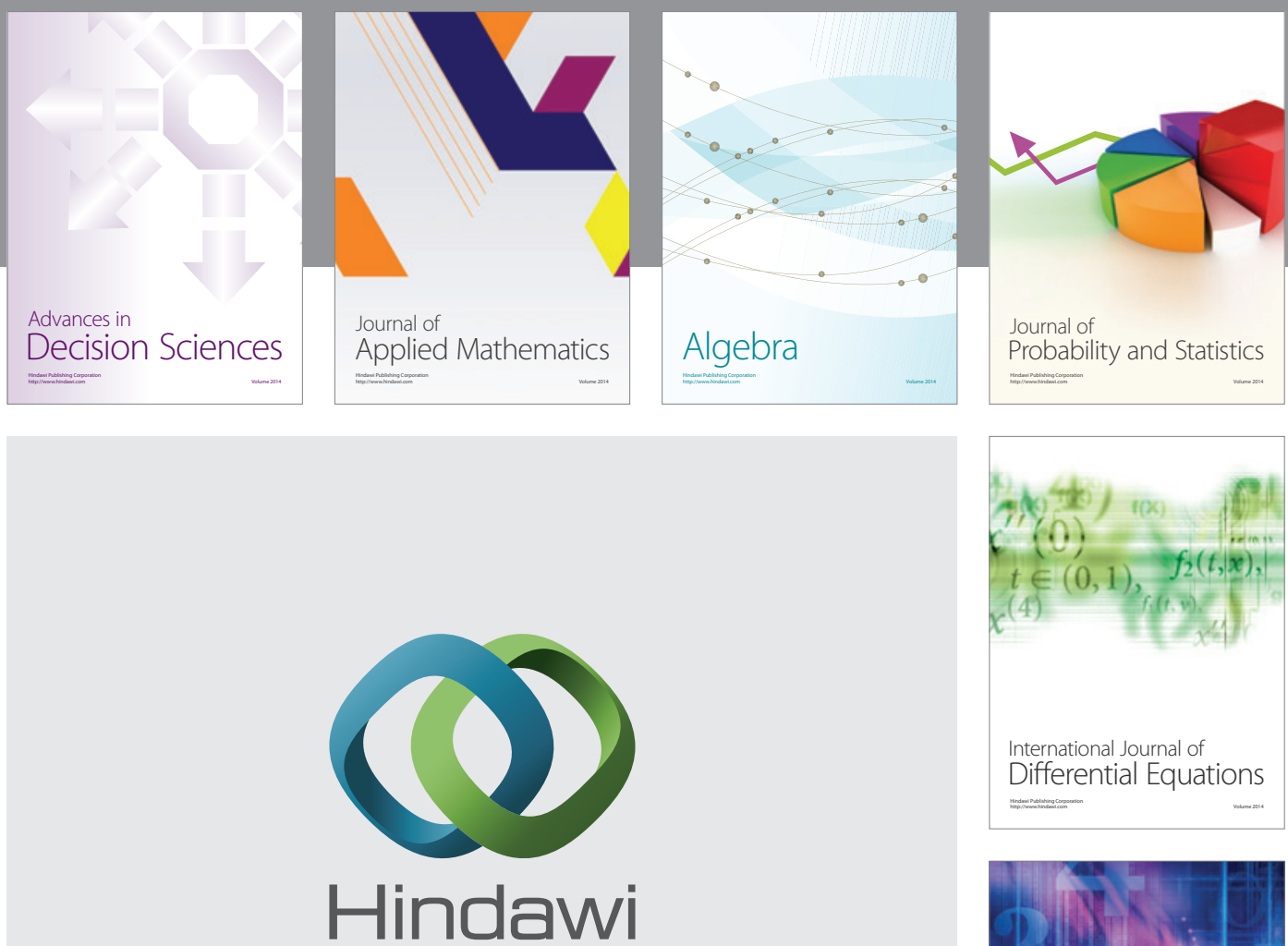

Submit your manuscripts at http://www.hindawi.com
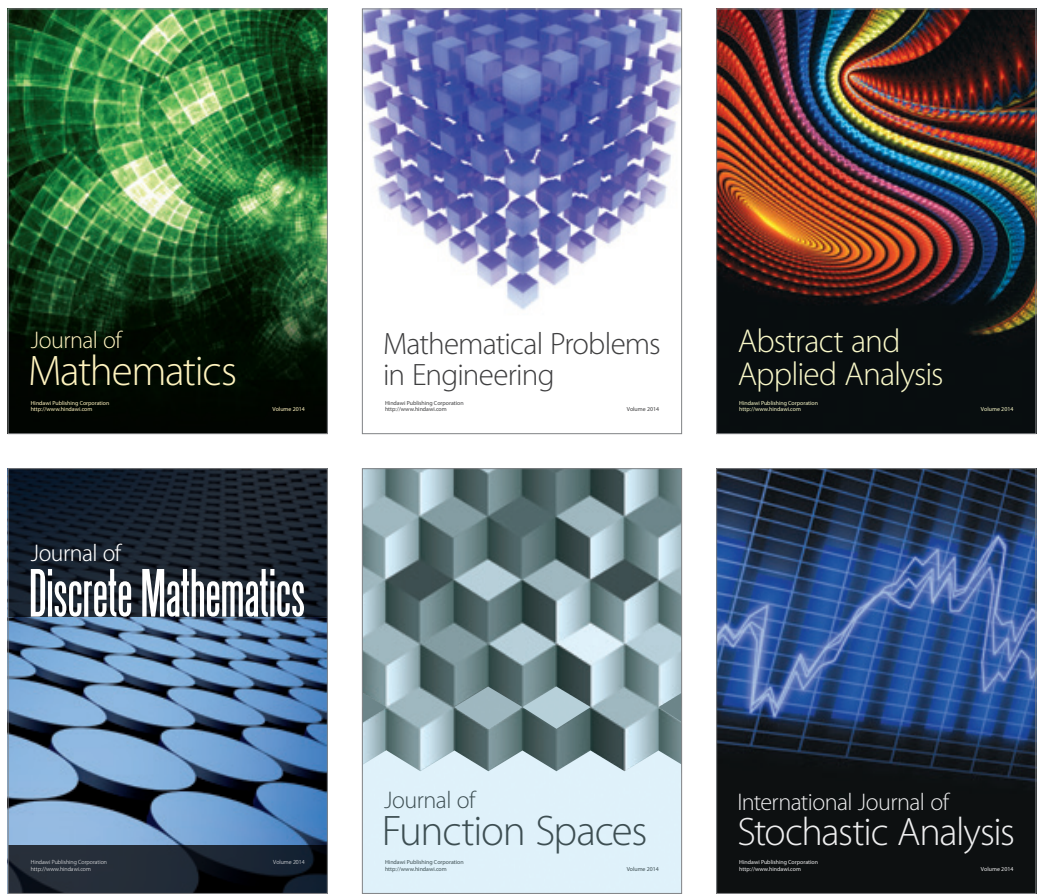

Journal of

Function Spaces

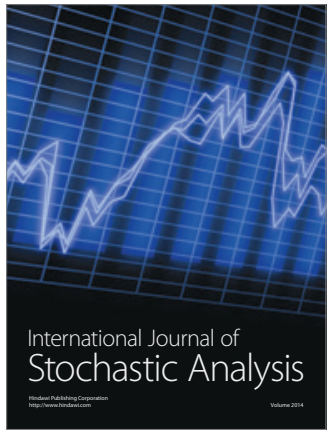

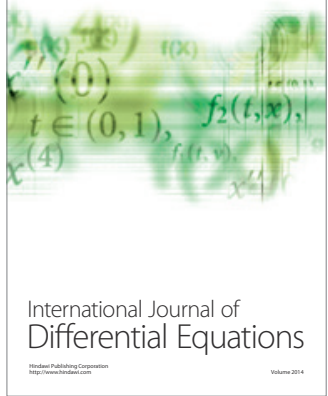
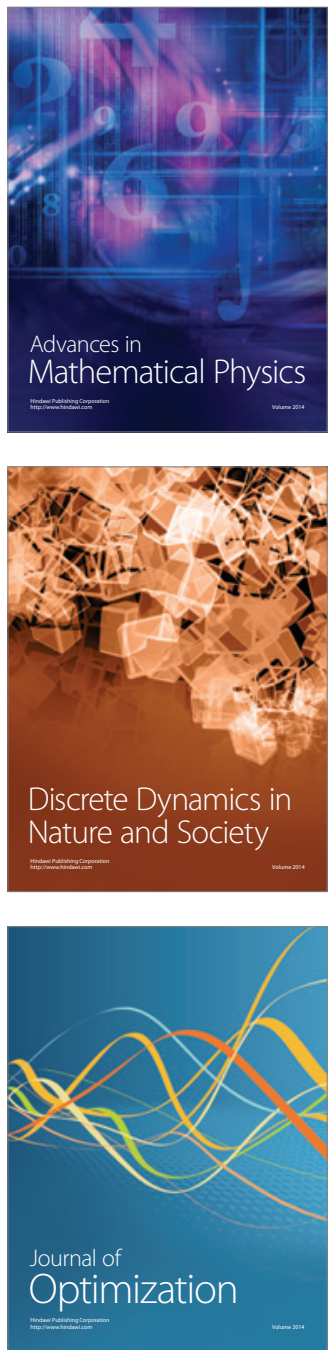